\title{
Automatic Genomics: a user-friendly program for the automatic designing and plate loading of medium- throughput qPCR experiments
}

\author{
Sergio Callejas*, Rebeca Alvarez*, and Ana Dopazo \\ Genomics Unit, Centro Nacional de Investigaciones Cardiovasculares (CNIC), Madrid, Spain
}

BioTechniques 50:46-50 (January 2011) doi 10.2144/000113574

Keywords: quantitative PCR; automation; liquid handing robot; experimental design

Supplementary material for this article is available at www.BioTechniques.com/article/113574.

${ }^{*}$ S.C. and R.A. contributed equally to this work.

Quantitative PCR (qPCR) remains the method of choice for gene and microRNA (miRNA) expression studies. Many laboratories wish to automate some or all of the steps of medium-throughput qPCR experiments through the use of various types of liquid handling robots. However, it is not uncommon to find cases in which scripts provided by the robot supplier are too rigid for user-specific applications, do not include all the desired options, or are too complicated to be modified by a nonprofessional programmer. Here, we present Automatic Genomics, a program that allows users with a limited programming background to automate medium-throughput qPCR experiments by using commercially available liquid-handling robots. The user is able to optimize the plate design in terms of number of genes, number of samples, and controls.

Despite improvements in established technologies such as expression microarrays and the emergence of new technologies such as RNA-seq $(1,2)$, quantitative PCR (qPCR) continues to be the method used by many researchers to study gene and microRNA (miRNA) expression or to validate results obtained with other technologies such as microarrays or deep sequencing (3). qPCR is a relatively easy-to-understand technology and does not require particularly expensive or complex equipment. These characteristics make qPCR experiments accessible to most laboratories and core facilities. However, from our experience as a genomics unit, researchers face two key problems when carrying out medium-throughput qPCR experiments: (i) defining an optimal experimental design and (ii) automating the process when the number of gene and sample combinations is too high to handle manually.

Optimal qPCR experimental design and the necessary controls have been described elsewhere (4-6). There are also several programs available for qPCR data analysis, including statistical analysis (5,7-10). However, to our knowledge, there has been no program available that has the flexibility to allow users to design 384-well plates for medium-throughput qPCR experiments and to generate all the necessary commands for a liquid handling robot to load the plate according to the user's instructions. Often, scripts supplied by the manufacturers of liquid-handling robots are too rigid or too specific for a particular protocol and allow little, if any, variation by users. Users can try to create a new script that considers all the necessary variables for a specific protocol, but this is often time-consuming and requires substantial programming skills. Consequently, it is not uncommon to find underused or even unused liquid-handling robots in laboratories. Many researchers wishing to carry out medium-throughput qPCR would be helped by a flexible software tool that allows them to automatically redesign experiments according to different variables (number of genes, samples, controls, etc.) and to define patterns for robotic loading of samples onto a 384-well plate.

Automatic Genomics ( $\mathrm{AG}$ ) is an application based on Microsoft Excel (Redmond, WA, USA) with a Visual Basic (VB) interface that allows any user with a limited programming background who has a Tecan Freedom EVO series robot (Tecan, Männedorf, Switzerland) to design 384-well plates for qPCR and create all the necessary commands for the robot to load them. Tecan, along with other companies such as Beckman Coulter (Brea, CA, USA), Eppendorf (Hamburg, Germany) with EpMotion, and Hamilton (Bonaduz GR, Switzerland), is one of the leading global providers of liquid-handling robots. All parameters required to design the 384-well plates for qPCR are introduced by the user through the VB interface. No knowledge of Microsoft Excel is required, although a minimum knowledge of Evoware software (Tecan) is required in order to make AG compatible with each user-worktablespecific configuration. The AG application allows the user to include all the controls and parameters needed to perform a mediumthroughput qPCR experiment, according to the most recently published standards of the scientific community (11).

Several subapplications help the user to automate qPCR-related steps, such as sample transfer, reverse transcription, and volume calculations according to userdefined parameters. Additionally, users can enter gene and sample names, either by typing or by file import, and AG will automatically generate an output file that can be directly imported into one of the 
A

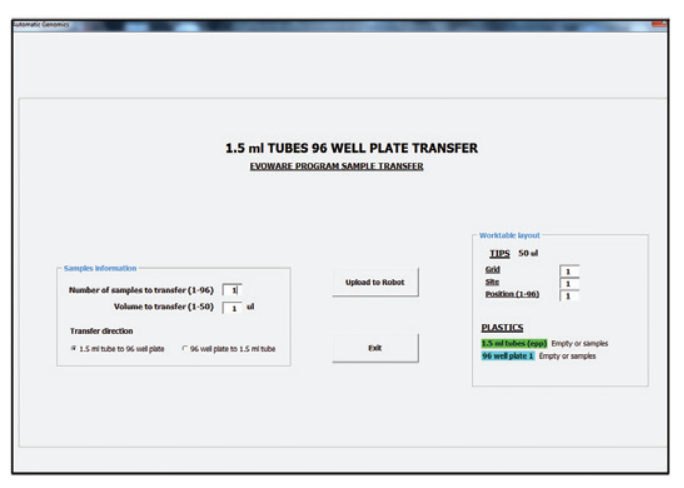

C

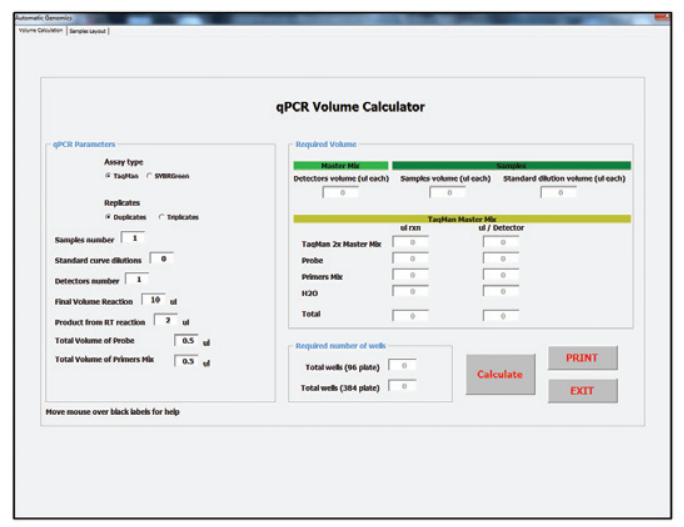

B

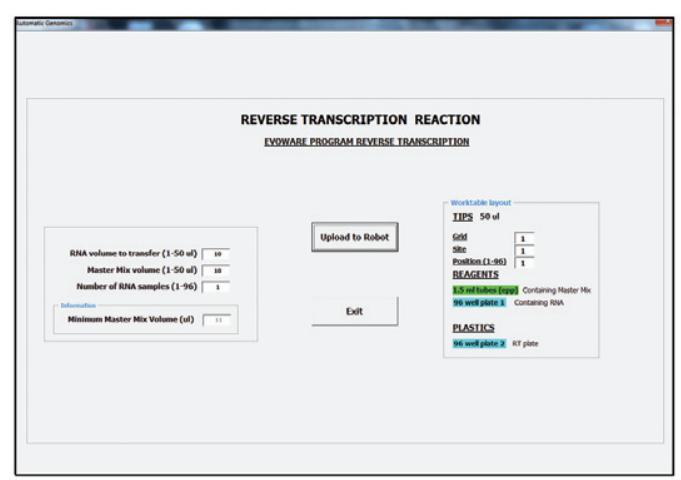

D

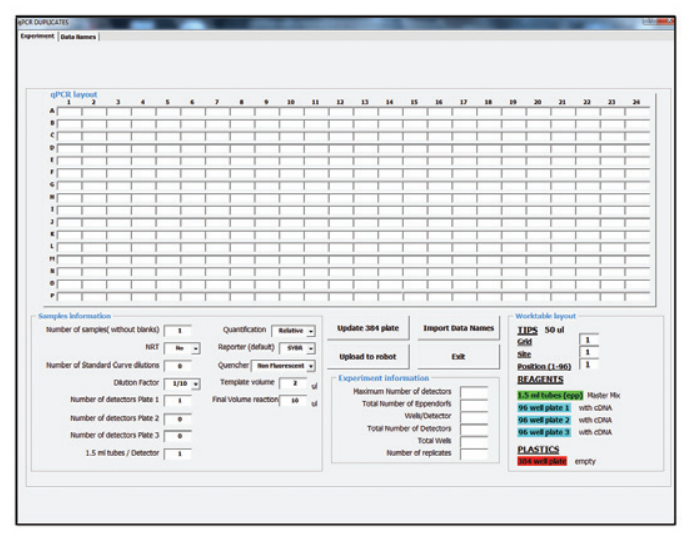

Figure 1. Four of the eight submenus included in AG. (A) A 1.5-mL tube/96-well plate sample transfer submenu. This submenu allows users to generate all necessary worklists for transfer of samples from 1.5-mL tubes to 96 -well plates and vice versa. (B) Reverse transcription reaction submenu. This submenu allows users to distribute the reverse transcription master mix and RNA samples to a 96-well plate for the reverse transcription reaction. (C) Exemplary volume calculator submenu (Std QPCR). This submenu calculates the required volume of every reagent needed for the qPCR. (D) Exemplary plate-loading submenu (Std qPCR). This submenu allows users to design the final layout of the 384-well plate before sending the information to the robot.

most widely used qPCR instruments, the ABI PRISM 7900HT Sequence Detection System (Applied Biosystems, Foster City, CA, USA). If used, this option significantly reduces the time-consuming step of preparing a template for the thermal cycler. AG can be used with any 384-well plate and any $\mathrm{qPCR}$ reader; however, the template for the qPCR reader has to be generated manually unless the ABI PRISM 7900HT is used.

In summary, the AG application allows any user with a limited knowledge of programming to skip the liquid-handling robot script-creation step for qPCR experiments, while retaining the required flexibility during the design of the experiment.

\section{Materials and methods}

The AG workflow is based on three steps: (i) specification of values for the variables, (ii) data calculation, and (iii) generation of output files. User intervention is only required in the first step, in which specific values for the different variables (number of genes, samples, replicates, controls, etc.) are introduced through the VB interface. Data calculation takes place automatically in hidden Microsoft Excel sheets; finally, output files (worklists) containing commands for the Tecan robot are generated and stored in a specific folder. To execute these worklists, the user only has to run the appropriate Evoware script included in the AG application package.

The AG package, together with tutorial videos demonstrating proper AG installation and worktable configuration, can be downloaded from the CNIC Genomics Unit web site (www.cnic.es/unidades/genomica/ automaticgenomics).

Robot and worktable requirements AG application has been developed for Tecan Freedom EVO series robots with a liquid-handling arm ( $\mathrm{LiHa}$ ) and disposable tips, regardless of the number of pins. AG is compatible with any worktable so long as it contains four positions for 96-well plates, one position for 384-well plates, 1-96 positions for $1.5-\mathrm{mL}$ tubes, and several $50-\mu \mathrm{L}$ disposable tip positions. Supplementary Figure $S 1$ shows an example of the intended robot worktable layout.

\section{Microsoft Excel and VB scripts}

The AG application includes a total of eight Microsoft Excel core files and one Microsoft Excel file containing VB user forms. Most of the eight Microsoft Excel core files generate worklists for different protocols: SampleTransfer allows transfer of up to 96 samples from $1.5-\mathrm{mL}$ tubes to a 96-well plate or vice versa. ReverseTranscription allows dispensing of a reverse transcription master mix into different RNA samples located in a 96-well plate. qPCRVolCalculator and miqPCRVolCalculator calculate the required volumes of reagents needed for gene expression in qPCR or miRNA qPCR (miqPCR), respectively. Std $q P C R 2 R$ and $S t d q P C R 3 R$ design and load 384-well plates for gene expression qPCR using duplicates or triplicates, respectively. $m i R N A P C R 2 R$ and miRNAPCR $3 R$ design and load 384-well plates for duplicate or triplicate miqPCR, respectively. The AutomaticGenomics file contains the VB interface and the main menu that will guide the user to all other applications. Once information about the experiment is introduced by the user through the VB interface and calculations have finished, output files containing all necessary instructions for the robot are generated and saved in a specific folder.

\section{Evoware scripts}

Evoware scripts have to be imported into the Evoware software in order to execute the worklists generated by Microsoft Excel core files. AG includes three Evoware 


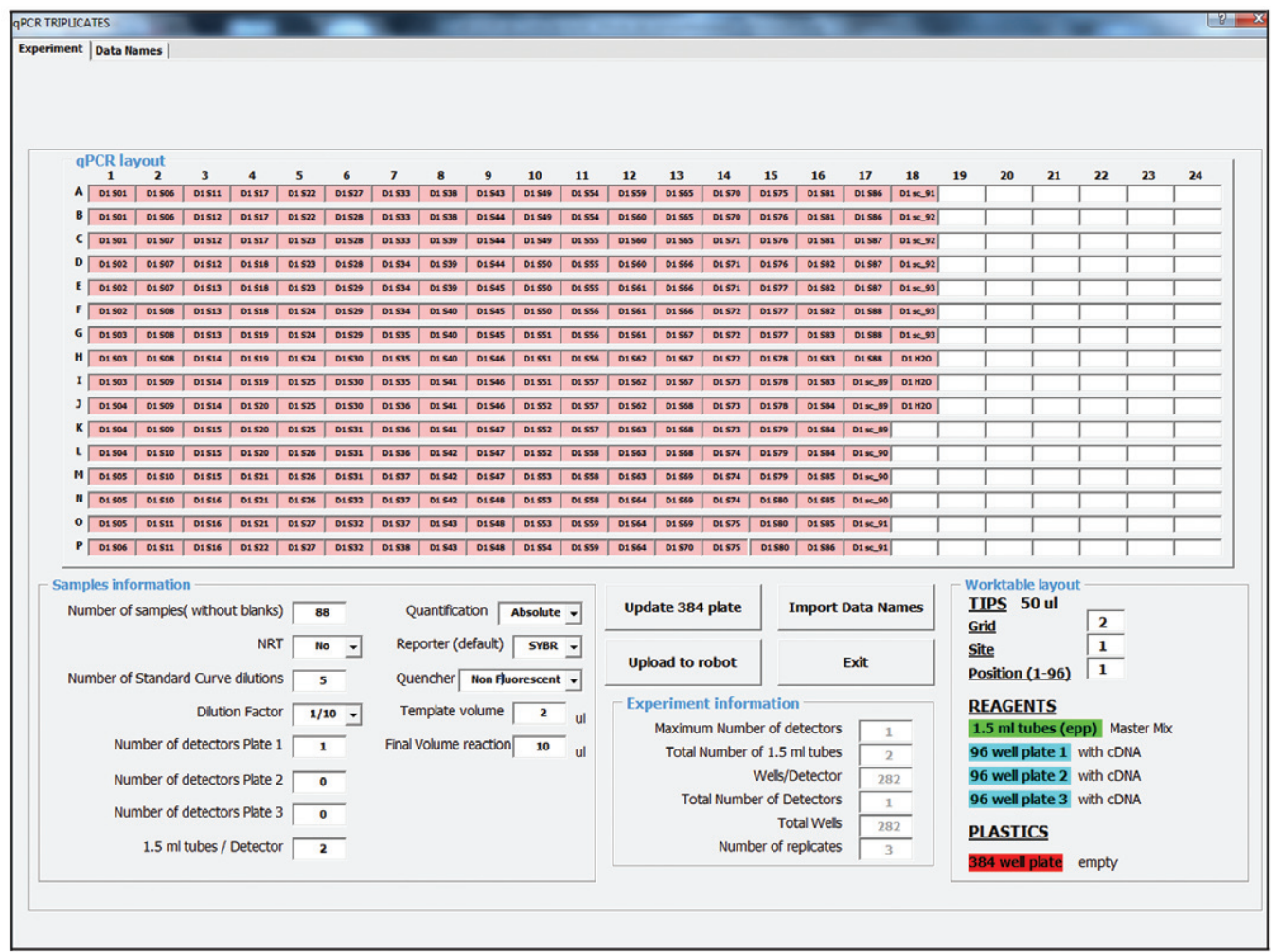

Figure 2. Exemplary qPCR plate-loading submenu. The upper part shows the sample layout that the robot will use to load the 384-well plate. The lower part displays parameters corresponding to the user-designed experiment. Users can easily define experimental variables by typing values into the appropriate text boxes. The information is sent to the robot by clicking Upload to Robot.

scripts: SampleTransfer executes the $1.5-\mathrm{mL}$ tubes/96-well plate transfer worklist, ReverseTranscription executes the reverse transcription worklist, and $q P C R$ executes worklists corresponding to the four different types of qPCR allowed in AG.

Liquid classes, carriers, and labware Liquid classes determine how the robot aspirates and dispenses liquids depending on several parameters such as viscosity or volume. A file containing a definition for all the liquid classes used by AG is downloaded together with the AG application package and has to be imported into the Evoware software. The user should use these liquid classes as a starting point; however, depending on each laboratory's conditions (temperature, humidity, etc.), they may need to be adjusted to ensure accurate aspirating and dispensing of liquids. Supplementary Table $S 1$ indicates which liquid class is used in each step of the various protocols.

Worktables for the three Evoware scripts are imported by the user as empty worktables, and carriers (removable supports that hold labware) and labware (disposable items such as microplates or sample tubes) have to be created and properly named in order to make AG compatible with each user worktable.

\section{cDNA samples}

Eighty-eight cDNA samples from breast cancer cell lines and tumors were used to validate the 384-well plate-loading process and to determine the variation between technical replicates. Also, five 1:10 cDNA dilutions were included to calculate technical variation at different concentrations. Three different genes (detectors) were studied in this experiment. Information about samples and qPCR conditions can be found in the Supplementary Materials.

\section{Results and discussion}

Once the robot worktable has been configured, the Evoware scripts installed, and all files downloaded and properly installed, the user can access the AG main menu. This menu allows the user to navigate through the eight submenus in AG according to the protocol of interest (Figure 1).

The Sample Transfer submenu allows transfer of 1-96 samples from $1.5-\mathrm{mL}$ tubes to a 96-well plate or vice versa. The user is asked for the number of samples to transfer, the volume to transfer, and the transfer direction. Once these data have been introduced, the Upload to Robot option can be selected to automatically generate the worklists. Information shown on the right part of each submenu indicates where reagents and empty labware must be located on the robot worktable. To execute the worklists, the user has only to open the Evoware software and run the SampleTransfer script.

The RT Reaction submenu allows transfer (if needed) of defined volumes of stock RNA samples from a 96-well plate to an empty 96 -well plate where reverse transcription will take place. The user can enter the master mix volume to be added to each RNA sample and the number of wells to which master mix will be dispensed. Once data have been uploaded to the robot, the user simply needs to run the ReverseTranscription Evoware script to execute the worklists.

The qPCRVolCalculator and miqPCRVolCalculator submenus will not generate any worklists. They are only intended to provide information about the volume required for qPCR samples (cDNA), qPCR master mix (containing all the required reagents except cDNA), and dilution volumes needed to build standard curves (standard curves can be used to calculate primer efficiency or to run absolute quantifications). The user is asked to define variables such as the type of assay to be carried out (SYBRGreen or TaqMan probes), the number of technical replicates to be loaded 
A

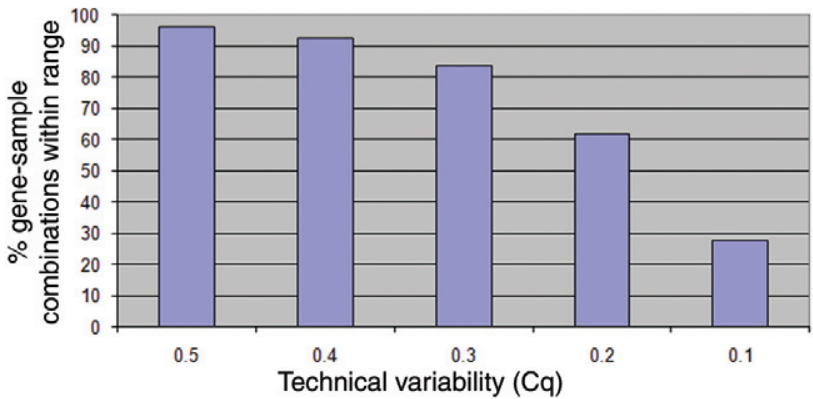

C

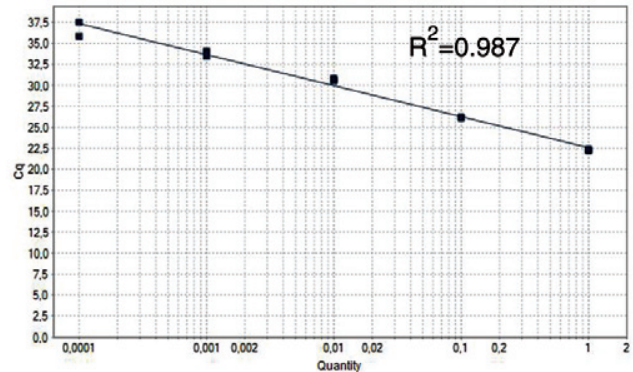

B

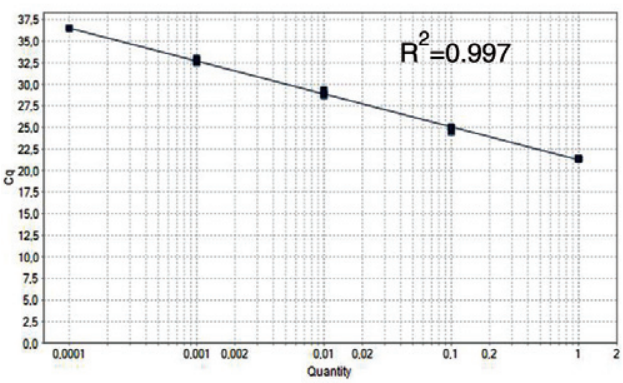

D

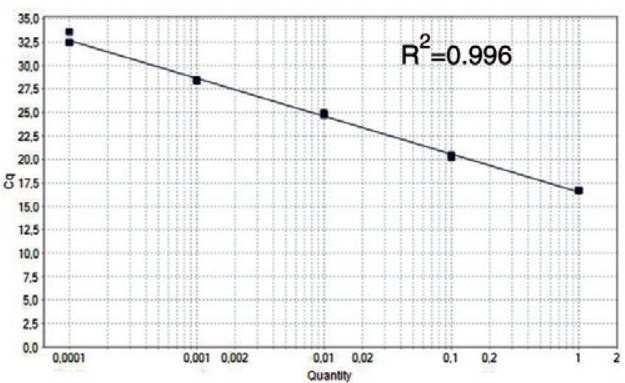

Figure 3. Quality control of automatically loaded plates. All gene-sample combinations were loaded in triplicate in a qPCR 384-well plate. (A) Percentage of gene-sample combinations that pass the indicated technical reproducibility cutoffs. The graph confirms high reproducibility between technical replicates, with more than $90 \%$ of the gene-sample combinations having a technical variation $<0.4 \mathrm{Cq}$. (B-D) Standard curves for the three genes included in the validation. Standard curves were built using five 1:10 cDNA dilutions. The $x$ axis represents the dilution factor for the cDNA template used in the qPCR. Technical variation remains very low even in the most diluted samples, as shown by the $R^{2}$ values.

(duplicates and triplicates are allowed), the number of samples, the number of dilutions to build a standard curve, and the number of genes to include in the plate. The final reaction volume and CDNA volume to be added can also be modified. Finally, the volume of primers or of the TaqMan assay can be entered to calculate the volume of each component of the master mix. Information about the experiment can be changed at any time and calculations updated by clicking Calculate. These two submenus contain a second page called Sample layout, in which the user can see the expected layout of cDNA samples and cDNA dilutions (if any) in a 96-well plate.

AG includes four submenus for designing and loading qPCR 384-well plates, allowing duplicate or triplicate determinations of Std qPCR and miqPCR: Std qPCR 2R, Std qPCR 3R, miRNA $q P C R 2 R$, and miRNA qPCR $3 R$. An example plate design screen is shown in Figure 1D. In these submenus, the user is asked to enter information about variables such as sample number, the inclusion of $N o$ Reverse Transcription samples (NRT; used as negative controls for genomic DNA contamination), the number of standard curve dilutions, and the dilution factor. Information about the number of genes must also be provided. Note that samples located in up to three 96-well plates can be loaded in the same 384-well plate, using either the same or different detector master mixes. This is particularly useful when the user has two or three small experiments they would like to load in a single 384-well plate. Another important feature is that each master mix can be divided among several $1.5-\mathrm{mL}$ tubes if the volume is too large for a single tube.

Users also have the option to provide information about the type of quantification (absolute/relative), the reporter, and the quencher. The names of the detectors and samples can either be typed or imported from a file. With this information, $\mathrm{AG}$ can generate the required input file for the Applied Biosystems 7900 qPCR instrument, and the generated input file can be directly imported into and run with the Applied Biosystems software.

There are no obvious differences at user level between the Std qPCR and the miRNA qPCR submenus. However, whereas $S t d q P C R$ includes just one reverse transcription for each cDNA, in miqPCR, there is a separate reverse transcription for each sample-gene combination. Consequently, these submenus differ radically in the mode of cDNA sample transfer from 96-well plate to 384-well plate. The miqPCR submenu should only be used for protocols requiring separate reverse transcription for each sample-gene combination; otherwise, select the Std qPCR submenu, even for miRNA expression analysis. For details about these differences see the tutorial videos (downloaded together with the AG package).

To validate the $q P C R$ volume calculator and $q P C R$ designer submenus, we tested sample distribution and technical reproducibility for three genes with $88 \mathrm{cDNA}$ samples from breast cancer cell lines and tumors. Five 1:10 cDNA dilutions were included to build standard curves and calculate technical reproducibility at different concentrations. qPCR was carried out using FAM-labeled probes in a $10-\mu \mathrm{L}$ final volume containing $2 \mu \mathrm{L}$ cDNA. Each sample-gene combination was loaded in triplicate.

Appropriate values for this experiment were entered into the $q P C R V$ olCalculator form, and the required volumes calculated. Note that a total of 846 wells is needed for this number of detectors, samples, and replicates. To optimize design, we used one detector per plate, so that each plate contained all samples; therefore, the whole experiment consisted of three 384-well plates. The following volumes were calculated: PCR master mix $(2481.6 \mu \mathrm{L})$, sample volume $(10 \mu \mathrm{L})$, and standard dilution volume $(10 \mu \mathrm{L})$. Since the required volume of each detector master mix was larger 
than $1.5 \mathrm{~mL}$, this volume was divided between two $1.5-\mathrm{mL}$ tubes (containing $1240.8 \mu \mathrm{L}$ each). To prepare the 96-well plate containing samples and cDNA dilutions, we followed the layout displayed on the Sample layout page (Supplementary Figure S2). This layout is recalculated by clicking Calculate after changing any value on the Volume calculation page. In the 96-well plate, the 88 cDNA samples are sorted by columns and are followed by the five dilutions for standard curves. This sample layout is the same for each of the three 384-well plates.

Once the detector master mixes and the 96-well sample plate were prepared, each 384-well plate was designed using the $q P C R 3 R$ menu. No NRT controls were included in this experiment, since the absence of genomic DNA had been previously checked (data not shown). Since each master mix was split between two tubes, the number of $1.5-\mathrm{mL}$ tubes/ detector was set at 2. Quantification was set to absolute in order to generate an ABI PRISM 7900HT input file with standards for dilution curves (relative quantification does not allow inclusion of samples as standards). When Update 384 plate is selected, the qPCR layout is shown in the upper part of the screen (Figure 2). Sample and detector names (Sample 01 to Sample 88 and Detector 01 to Detector 03 , respectively) can be typed or imported into the Data names page. At this point, users can adjust the settings and check the new 384-well plate design by clicking Update 384 plate. When Upload to Robot is selected, the worklists are generated. At this stage, the two $1.5-\mathrm{mL}$ tubes, the 96-well sample plate, and the empty 384-well plate are positioned on the robot worktable. Positions for each labware item are indicated in the lower right part of the screen on the $q P C R$ menus.

The Evoware program was opened, and the qPCR script run. This script executes work lists generated by AG. The robot loads the detectors and samples in the exact position shown in $q P C R$ layout. The input file for the ABI PRISM 7900HT sequence detection system (SDS) is stored in the $S D S$ subfolder in the Automatic Genomics folder. To optimize the process, Evoware calculates the optimum number of tips to be used to distribute the PCR master mixes. The PCR master mix is aspirated automatically with several tips (1-8 tips) and then dispensed in the 384-well plate using the multidispensing option. cDNA samples are distributed using all the necessary tips in a single-aspiration and single-dispense loop.
Once the 384-well plates were loaded, the generated SDS files were directly imported into the ABI PRISM 7900HT Real-Time PCR System, and the 384-well plates were run on a standard cycling protocol. To check technical reproducibility, Cq values were automatically extracted using the SDS 2.3 software (Applied Biosystems), and the data were then imported into qBasePlus (5). Among many other features, $\mathrm{qBasePlus}$ can run a quality control check of technical replicates. The graph in Figure 3A indicates the percentage of the 282 sample-gene combinations (including cDNA dilutions) that pass different quality control cutoffs for technical reproducibility (the maximum difference allowed between any three technical replicates). At a cutoff of 0.5 cycles, $96 \%$ of all sample-gene combinations passed the quality control, and at a cutoff of 0.2 cycles, $61.5 \%$ of sample-gene combinations were still within the limit. Based on our experience, this reproducibility is very high and is certainly higher than that achieved when loading by hand (Supplementary Figure S3). Standard curves for the three genes also showed high technical reproducibility over different concentrations, with $R^{2}=0.997,0.987$, and 0.996 (Figure 3, B-D).

This report describes the validation of AG as an easy-to-use application for nonexpert programmers who need to run medium-throughput qPCR experiments. AG can design (or redesign from an existing pattern) the layouts for 384-well plates used in qPCR experiments and then accurately load those plates, thereby saving time, and reducing human error. We believe AG will be particularly useful for laboratories and core facilities wishing to automate qPCR experiments but who lack the support of an expert programmer.

\section{Acknowledgments}

The authors thank Maria Garcia and Ana Osorio (Spanish National Cancer Centre, CNIO, Madrid, Spain) for the breast cancer cell lines and tumors used for validation purposes and for their feedback and comments during the set up and adjustments of Automatic Genomics and Fátima Sánchez Cabo (CNIC) for support and suggestions. We also thank Simon Bartlett (CNIC) for English editing.

\section{Competing interests}

The authors declare no competing interests.

\section{References}

1.Marioni, J.C., C.E. Mason, S.M. Mane, M. Stephens, and Y. Gilad. 2008. RNA-seq: an assessment of technical reproducibility and comparison with gene expression arrays. Genome Res. 18:1509-1517.

2.Wang, Z., M. Gerstein, and M. Snyder. 2009. RNA-Seq: a revolutionary tool for transcriptomics. Nat. Rev. Genet. 10:57-63.

3. VanGuilder, H.D., K.E. Vrana, and W.M. Freeman. 2008. Twenty-five years of quantitative PCR for gene expression analysis. BioTechniques 44:619-626.

4. Nolan, T., R.E. Hands, and S.A. Bustin. 2006. Quantification of mRNA using real-time RT-PCR. Nat. Protocols 1:15591582.

5.Hellemans, J., G. Mortier, A. De Paepe, F. Speleman, and J. Vandesompele. 2007. qBase relative quantification framework and software for management and automated analysis of real-time quantitative PCR data. Genome Biol. 8:R19.

6. Derveaux, S., J. Vandesompele, and J. Hellemans. 2010. How to do successful gene expression analysis using real-time PCR. Methods 50:227-230.

7. Pabinger, S., G.G. Thallinger, R. Snajder, H. Eichhorn, R. Rader, and Z. Trajanoski. 2009. QPCR: application for real-time PCR data management and analysis. BMC Bioinformatics 10:268.

8.Pfaffl, M.W., G.W. Horgan, and L. Dempfle. 2002. Relative expression software tool (REST) for group-wise comparison and statistical analysis of relative expression results in real-time PCR. Nucleic Acids Res. 30:e36.

9. Muller, P.Y., H. Janovjak, A.R. Miserez, and Z. Dobbie. 2002. Processing of gene expression data generated by quantitative real-time RT-PCR. BioTechniques 32:13721379.

10.Jin, N., K. He, and L. Liu. 2006. qPCR-DAMS: a database tool to analyze, manage, and store both relative and absolute quantitative real-time PCR data. Physiol. Genomics 25:525-527.

11.Bustin, S.A., V. Benes, J.A. Garson, J. Hellemans, J. Huggett, M. Kubista, R. Mueller, T. Nolan, et al. 2009. The MIQE guidelines: minimum information for publication of quantitative real-time PCR experiments. Clin. Chem. 55:611-622.

Received 5 August 2010; accepted 28 October 2010.

Address correspondence to Sergio Callejas, Genomics Unit, Centro Nacional de Investigaciones Cardiovasculares (CNIC), Melchor Fernández Almagro 3, 28029 Madrid, Spain.e-mail: scallejas@cnic.es

To purchase reprints of this article, contact: carmelitag@fosterprinting.com 